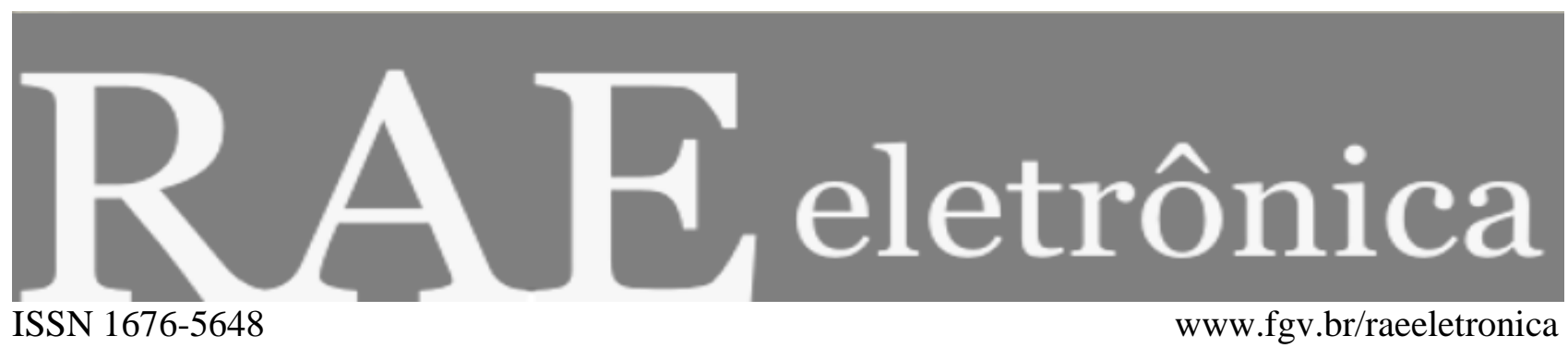

\title{
ARTIGOS
}

\section{ANÁLISE DA CAPACIDADE EXPLICATIVA DO INVESTIMENTO PELO “q” DE TOBIN EM EMPRESAS BRASILEIRAS DE CAPITAL ABERTO}

ANALYSIS OF THE EXPLANATORY CAPACITY OF INVESTMENT USING TOBIN'S “q” IN

\section{BRAZILIAN PUBLICLY QUOTED COMPANIES}

\section{Edson Luis Kammler}

Coordenador do Curso de Ciências Contábeis da Faculdade Concórdia - SC, Brasil

edsonkammler@hotmail.com

\section{Tiago Wickstrom Alves}

Professor do Departamento de Ciências Econômicas da Universidade do Vale do Rio dos Sinos - RS, Brasil

twa@unisinos.br

Recebido em 11.12.2007. Aprovado em 21.07.2009. Disponibilizado em 25.09.2009

Avaliado pelo sistema double blind review

Editor Científico: Ricardo Ratner Rochman

RAE-eletrônica, v. 8, n. 2, Art. 12, jul./dez. 2009.

http://www.rae.com.br/eletronica/index.cfm?FuseAction=Artigo $\& I D=4769 \&$ Secao=ARTIGOS $\&$ Volume $=8 \&$ Numero=2 $\&$ $\mathrm{Ano}=2009$

CCopyright 2009 FGV-EAESP/RAE-eletrônica. Todos os direitos reservados. Permitida a citação parcial, desde que identificada a fonte. Proibida a reprodução total. Em caso de dúvidas, consulte a Redação: raeredacao@fgv.br; 55 (11) $3799-7898$.

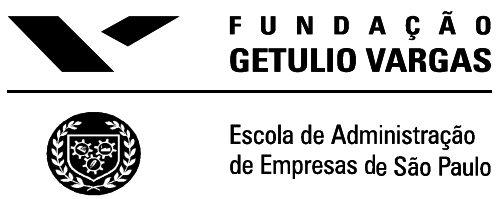




\section{RESUMO}

Este trabalho teve como objetivo testar a capacidade de explicação dos investimentos pela teoria do "q" de Tobin nas empresas brasileiras de capital aberto. Fez-se o estudo através de uma análise com dados em painel, utilizando dados do Balanço Contábil e cotações das ações na Bolsa de Valores. A maior dificuldade da teoria do "q" de Tobin é a apuração do custo de reposição, porém existem aproximações que podem facilitar o cálculo. Neste trabalho, foram adotados os modelos propostos por Chung e Pruitt (1994) e por Lee e Tompkins (1999), por serem considerados uma aproximação simplificada do "q". Para essas análises foram utilizados os anos de 2002 a 2008, utilizando a variável "q" no mesmo ano do investimento, defasado em um período e ao quadrado. Este trabalho inova ao evidenciar possibilidades de medir o custo de reposição do capital e, com isso, testar o "q" de Tobin para o Brasil. Conclui-se que é possível explicar a variação dos investimentos através do "q" de Tobin, obtendo resultados mais significantes do que outras análises que usaram outras aproximações para o "q" de Tobin.

PALAVRAS-CHAVE “q" de Tobin, investimento, decisão, demonstrações financeiras, custo de reposição de capital.

\section{ABSTRACT}

The aim of this work is to test the explanatory capacity of investments in publicly-quoted Brazilian companies using Tobin's “ $q$ ” theory. The study was carried out by means of panel data analysis, using data taken from the balance sheet and share prices on the stock exchange. The greatest difficulty with Tobin's “ $q$ " theory is calculating the replacement cost of capital, although there are approximations that can facilitate the calculation. In this work we adopted the models proposed by Chung and Pruitt (1994) and by Lee and Tompkins (1999), because they were considered to be a simplified approximation of " $q$ ". For these particular analyses we worked with the years 2002 to 2008, using the " $q$ " variable in the same year as the investment, with a one period lag and squared. This work is innovative in that it provides evidence of the possibility of measuring the replacement cost of capital and, therefore, testing Tobin's " $q$ " theory within the Brazilian context. We concluded that it is possible to explain variations in investments by means of Tobin's " $q$ " theory, thereby obtaining more significant results than other analyses that used other approximations for Tobin's " $q$ ".

KEYWORDS Tobin's “ $q$ ”, investment, decision, financial statements, replacement cost of capital. 


\section{INTRODUÇÃO}

A determinação dos gastos de investimento é uma das questões teóricas mais complexas e controversas. Decisões de investir envolvem considerações sobre preços, produtos e tecnologias tanto correntes como futuros, sobre taxas de utilização da capacidade produtiva, sobre comportamento de outros empresários, sobre perspectivas políticas, entre outras. Dessa característica resulta o fato de que decisões políticas relativas aos ciclos econômicos ou ao crescimento econômico são baseadas em informações duvidosas e carentes de evidência empírica sobre as causas das variações do investimento (ANDRADE, 1987, p. 75).

Uma dificuldade para explicar as variações dos investimentos é o fato de que diferentes fatores determinam diferentes tipos de gastos. Em função disso é que decorre a afirmação de Shapiro (1985, p. 269) de que nenhuma teoria única é capaz de ser aplicada a todas as formas de gastos com investimentos. Ainda, segundo Casagrande (2002a), o investimento é uma das mais importantes deliberações econômicas, uma vez que define o desempenho da economia. Porém, ele não é explicado adequadamente pelos modelos convencionais por não capturar os efeitos da incerteza.

Uma das teorias mais gerais e aceitas é a do "q" de Tobin. Ela considera que o investimento pode ser significativamente explicado pela relação existente entre o valor de mercado das empresas e o custo de reposição do capital. Diversos estudos foram feitos nos Estados Unidos e na Europa, confirmando a capacidade de explicação dos gastos com investimentos através do "q" de Tobin. Entretanto, poucos trabalhos foram realizados no Brasil para conferir o poder de explicação dessa teoria e, além disso, obtiveram resultados insatisfatórios. Crê-se que esses resultados decorram basicamente das proxis utilizadas no custo de reposição do capital.

Dois artigos sugerem uma nova metodologia para encontrar o "q" de Tobin. Partindo de dados contábeis, esses trabalhos estabelecem uma relação que, em diversos testes, resultaram em uma aproximação significativa do "q" de Tobin calculado pelo modelo clássico.

No que se refere à teoria do "q" de Tobin, Fama e Barros (2000) apresentam um levantamento amplo e interessante de artigos científicos que tratam do "q" aplicado a finanças. Outras pesquisas utilizam o "q" de Tobin para avaliar o capital intelectual de uma organização, como o de Paulo (2000); outras como um modelo de análise de balanço, como de Kassai, Kassai e Assaf Neto (2002). Isso evidencia o interesse e as potencialidades desse modelo que começou como um modelo de equilíbrio da teoria monetária.

No entanto, pretende-se inovar com a utilização de um teste do "q" de Tobin para o mercado 
brasileiro, utilizando dados contábeis como substitutos do custo de reposição do capital, segundo a metodologia proposta por Chung e Pruitt (1994) e Lee e Tompkins (1999) com dados em painel e modelando a relação do "q" com o investimento. Busca-se, assim, contribuir para a avaliação desse modelo para o entendimento do investimento.

\section{A TEORIA DO “q” DE TOBIN}

A teoria do "q" de Tobin surgiu de uma teoria monetária numa abordagem de equilíbrio geral. Tobin (1969), inicialmente, propôs um sistema de quatro equações para sua teoria monetária: uma para a definição da riqueza como a soma entre o valor real do estoque de capital existente e a oferta real de moeda, a razão "q", o preço de oferta de capital e a rentabilidade de retenção de moeda, considerando como variáveis exógenas o estoque de capital $K$, o estoque de moeda $M$, o produto $Y$, a eficiência marginal de capital $R$, a taxa esperada de inflação $\pi$ e a taxa nominal de juros r'm.

Nesse modelo, surgiu a razão "q" entre o valor de mercado da empresa e o custo de reposição de seus ativos. Se o valor de mercado for maior que o custo de reposição $(q>1)$, haverá retorno ao se investir; caso contrário, $(\mathrm{q}<1)$, a empresa não deveria fazer o investimento. Portanto, o investimento é uma função crescente da relação "q".

Para o cálculo do "q", o valor de mercado pode ser obtido, para empresas com ações negociadas na bolsa de valores, pela multiplicação do número de ações pela sua cotação. O maior problema apresentado nesse modelo é a apuração do valor de reposição dos ativos, que não podem ser mensurados facilmente. Para estimar o valor de reposição, é preciso ajustar os valores contábeis não apenas pela variação dos preços da economia, mas, também, pela variação tecnológica ocorrida no período e pela depreciação real dos ativos em oposição à depreciação contábil (FAMA e BARROS, 2000, p. 35).

Um estudo que tentou construir um método que aproximasse adequadamente o "q" teórico daquele estimado a partir de dados reais foi o de Lindenberg e Ross (1981). Segundo os autores, o conceito de custo de reposição é muito complexo e de difícil mensuração em razão da falta, em muitos casos, de um mercado ativo de bens de capital usados. Entretanto, eles calcularam o custo de reposição, dividindo os ativos da empresa em três grandes categorias: instalações e equipamentos, estoques e outros ativos, como caixa, títulos negociáveis e terrenos. Por suposição, os ativos da última categoria têm o custo de reposição aproximado ao valor contábil. A dificuldade é a mensuração do valor de 
reposição das instalações e equipamentos e dos estoques.

Lindenberg e Ross (1981) calcularam o valor de reposição dos ativos através da seguinte fórmula:

$$
R C=T A+R N P-H N P+R I N V-H I N V
$$

Em que:

$R C=$ Valor de reposição dos ativos

$T A=$ Ativo total (contábil)

$R N P=$ Valor de reposição das instalações e equipamentos

$H N P_{-}=$Valor histórico das instalações e equipamentos

$R I N V=$ Valor de reposição dos estoques

$H I N V=$ Valor histórico dos estoques

Para o valor de reposição das instalações e equipamentos, foi escolhida uma data arbitrária para o valor contábil assumido como o custo de reposição. No trabalho, o teste empírico envolvia o período de 1960 a 1977 e a data inicial escolhida foi 1952. A partir dessa data, o valor dos equipamentos e instalações do ano anterior foi corrigido por uma taxa de aumento no preço dos bens de capital, por uma taxa real de depreciação e por uma taxa de progresso técnico, a fim de obter o custo de reposição do ano atual dos bens, ao qual foram somadas as novas aquisições da empresa realizadas durante o ano em instalações e equipamentos.

Para o valor de estoques, Lindenberg e Ross (1981) argumentam que as empresas podem utilizar diferentes métodos de contabilização de estoques, como: primeiro a entrar - primeiro a sair (PEPS), último a entrar - primeiro a sair (UEPS) e média ponderada. Nesse caso, diferentes ajustes devem ser feitos de acordo com cada método. Se a empresa utilizar o PEPS, o valor atual do estoque seria uma aproximação razoável do seu custo de reposição, pois os produtos estariam contabilizados pelo seu preço mais recente. Portanto:

$R I N V_{t}=H I N V_{t}$

Se a empresa utilizasse o método UEPS, o valor contábil estaria abaixo do custo de reposição, pois os produtos estariam sendo contabilizados pelo preço mais antigo. Para o ajuste, os autores 
recomendam a utilização de um índice de correção de preços apropriado para estoques, através da seguinte fórmula:

$R I N V_{t}=R I N V_{t-1}\left(\frac{P_{t}}{P_{t-1}}\right)+\left(H I N V_{t}-H I N V_{t-1}\right)\left[\frac{0,5\left(P_{t}+P_{t-1}\right)}{P_{t-1}}\right]$

Em que:

$P=$ Índice de correção dos preços do estoque-

Se a empresa utilizar o método da média ponderada, os autores consideram que o estoque no tempo $t$ seria, aproximadamente, uma média dos valores entre o tempo $t-1$ e $t$. Nesse caso, recomendam a utilização da fórmula a seguir para o cálculo do custo de reposição:

$R I N V_{t}=H I N V_{t} \times \frac{2 P_{t}}{\left(P_{t}+P_{t-l}\right)}$

Em que:

$P=$ Índice de correção dos preços do estoque.

Um estudo alternativo para o cálculo do "q" de Tobin foi apresentado por Chung e Pruitt (1994). No seu trabalho, os autores apresentaram uma aproximação simplificada para o cálculo do "q", definida simplesmente como:

$q=\frac{V M+P S+V D}{A T}$

Em que:

$V M=$ Valor de mercado das ações das empresas-

$P S=$ Valor de mercado de uma espécie de títulos preferenciais emitidos pelas empresas americanas, que nos Estados Unidos pagam dividendos perpétuos

$V D=$ Valor do passivo circulante menos o ativo circulante somado ao valor contábil das dívidas de longo prazo

$A T=$ Valor contábil dos ativos totais da empresa 
Nessa equação, os autores utilizam quase que somente dados contábeis para o cálculo, os quais são facilmente encontrados nas demonstrações contábeis das empresas. Os valores referentes à cotação das ações podem ser obtidos na bolsa de valores.

No seu estudo, Chung e Pruitt compararam os resultados obtidos do "q" aproximado com os resultados de Lindenberg e Ross. A análise foi feita com dez diferentes amostras de empresas norteamericanas no período de 1978 a 1987. A menor dessas amostras tinha 1.201 empresas e a maior, 1.617. Os autores concluíram que, em nenhuma das 10 regressões, o coeficiente de explicação do "q" aproximado foi menor que 0,966, ou seja, no mínimo, 96,6\% do "q" calculado por Lindenberg e Ross pode ser explicado pelo "q" aproximado, assim, existe a possibilidade de se utilizar uma aproximação simplificada do "q" quando os dados necessários aos cálculos teoricamente mais corretos, como os de Lindenberg e Ross, não estão disponíveis.

Lee e Tompkins (1999) apresentaram uma interpretação alternativa para o "q" aproximado de Chung e Pruitt. A equação apresentada pelos autores pode ser escrita da seguinte forma:

$q=\frac{V M+P S+E L P+E S T+P C-A C}{A T}$

$V M=$ Valor de mercado das ações das empresas

$P S=$ Valor de mercado de uma espécie de títulos preferenciais emitidos pelas empresas americanas, que nos Estados Unidos pagam dividendos perpétuos

$E L P=$ Valor contábil do exigível a longo prazo

$E S T=$ Valor contábil dos estoques

$P C=$ Valor contábil do passivo circulante

$A C=$ Valor contábil do ativo circulante

$A T=$ Valor contábil dos ativos totais da empresa

Segundo Lee e Tompkins (1999), os últimos três termos do numerador refletem o valor das obrigações de curto prazo da empresa líquidos dos ativos de curto prazo. Uma questão que pode ser levantada é a liquidez do estoque, que, em muitos casos, não pode ser transformado em dinheiro imediatamente, dificultando o seu uso na liquidação das dívidas. Isso justificaria a retirada do mesmo no cálculo do numerador do "q".

Erickson e Withed (2000) testaram a relação entre investimentos e o "q" de Tobin através da 
utilização de dados em painel em 737 empresas industriais dos Estados Unidos com dados de 1992 a 1995. Segundo os autores, os testes que utilizam somente o "q" como variável explicativa dos investimentos apresentam baixos resultados, sendo que esse problema pode ser em decorrência de erros de medição, já que alguns dados são de difícil mensuração, como o custo de reposição dos ativos. Apesar disso, muitos estudos são realizados pela facilidade de aplicação do modelo e pelo poder explicativo do investimento pela teoria proposta. Sua conclusão foi que, apesar de o "q" ser uma teoria simples, não pode ser considerada como a "última palavra" sobre investimentos e que outros aspectos devem ser levados em conta, como restrição de capital e fluxo de caixa.

Um estudo brasileiro utilizando o "q" para avaliar o comportamento do investimento agregado é o de Andrade (1987), cujo período da análise compreendeu os anos de 1971 a 1985. No trabalho, a autora utilizou a soma do valor de mercado de todas as empresas para o numerador e como custo reposição foi utilizada a soma do valor do Patrimônio Líquido que foi considerado uma possível aproximação do custo de reposição do estoque de capital da amostra (ANDRADE, 1987, p. 113).

O estudo indicou que a razão "q" afeta, significativamente, o investimento agregado, mas no sentido inverso do esperado. Segundo a autora, os sinais negativos podem ter diversas explicações: podem indicar a ingenuidade do modelo de equilíbrio para representar situações de desequilíbrio, a economia poderia estar em desequilíbrio, mas dirigindo-se para o equilíbrio, situação em que o sinal voltaria a ser positivo. Outras explicações seriam a utilização de dados anuais (considerada pouco provável) e que o preço das ações pode não estar refletindo o valor de mercado das empresas.

Analisando a série de dados utilizados, foi possível verificar que a soma do patrimônio líquido é maior que o valor de mercado das empresas em $80 \%$ dos anos da análise, o que resulta num valor do “q" menor que 1. Segundo a teoria, se o "q" fosse menor que 1, a empresa teria que deixar de investir até chegar a um equilíbrio com o "q" igual a 1. Isso, de fato, não aconteceu, pois o investimento, na década de 1970, foi de aproximadamente $23 \%$ do PIB e de $18 \%$ na década de 1980 , bem acima dos 15\% dos anos de 2002 e 2003. Isso mostra que as ações das empresas poderiam estar sendo subavaliadas na bolsa de valores nos anos da análise em virtude da pouca significância do mercado acionário brasileiro.

\section{PROCEDIMENTOS METODOLÓGICOS}

O “q” de Tobin foi calculado, inicialmente, para o ano de 2002 através da seguinte fórmula: 
$q=\frac{V M+V D}{A T}$

Em que:

$V M=$ Valor de mercado das ações da empresa

$V D=$ Valor das dívidas da empresa, obtidos através do passivo circulante menos o ativo circulante somado ao valor contábil das dívidas de longo prazo

$\mathrm{AT}=$ Ativo total da empresa

Para o investimento, foi considerada a variação percentual de 2001 para 2002 do valor do ativo imobilizado mais os estoques, sendo que para a obtenção dos valores foram utilizados os balanços consolidados das empresas. Isso levaria à determinação do investimento líquido, que é aquele que aumenta o estoque de capital (SACHS e LARRAIN, 1995, p. 129) e que é a determinante nas teorias do investimento. Conforme Gordon (2000, p. 364), o investimento líquido (I) é a variação do estoque de capitais $(\Delta \mathrm{K})$ que ocorre em cada período, ou seja: $\mathrm{I}=\Delta \mathrm{K}=\mathrm{K}_{\mathrm{t}}-\mathrm{K}_{\mathrm{t}-1}$.

Embora o valor do investimento a ser identificado no Balanço Patrimonial das empresas seja uma questão controversa, que parece não ter consenso, é justificada porque o investimento em imobilizado consiste nos gastos das empresas em maquinário durável, em equipamentos e estruturas. Esses gastos representam a quase totalidade do valor do investimento total. Os estoques compreendem as matérias-primas, os bens semi-acabados no processo de produção e os bens acabados em poder das empresas, que também podem ser considerados como investimentos (SACHS e LARRAIN, 1995, p. 129; DORNBUSCH e FISCHER, 1982, p. 154).

As estimações foram realizadas com dados em Painel utilizando o software Eviews 6.0. A justificativa para a utilização desse procedimento decorre das especificidades de cada empresa em relação aos investimentos, então, uma estimação que não levar em conta essas especificidades $-C_{i}$ na equação 8 - terá um erro de composição $\left(u_{i t}=C_{i}+\varepsilon_{i t}\right)$, gerando um viés de heterogeneidade. $\mathrm{O}$ fato de $C_{i}$ não ter o subscrito "t" é justamente porque ele é constante ao longo do tempo. Segundo Wooldridge (2002, p. 247), de fato, a motivação primária para a utilização de Dados em Painel é para resolver o problema de variáveis omitidas.

Formalmente, a equação inicial a ser testada pode ser escrita da seguinte forma: 


$$
\operatorname{Inv} v_{i t}=\beta_{0}+\beta_{1} q_{i t}+C_{i}+T D C F_{t}+\mathcal{E}_{i t}
$$

Em que:

$I n v_{i t}=$ Taxa de investimento da empresa $i$ no ano $t$, construída de quatro formas, como segue:

$I M=$ Variação porcentual do imobilizado

$I M V=$ Variação, em valor, do imobilizado

$I N V=$ Variação porcentual do imobilizado mais estoques

$I N V V=$ Variação, em valor, do imobilizado mais estoques

$\beta_{0}=$ Investimento autônomo médio das empresas;

$\beta_{1}=$ Parâmetro que mede a sensibilidade do investimento para uma dada variação no q;

$q_{i t}=\mathrm{q}$ de Tobin da empresa $i$ para o ano $t$, calculado conforme a equação (7), construído de duas formas, como segue:

$q=$ Valor do q de Tobin conforme Chung e Pruit (equação 5)

$q e=$ Valor do q de Tobin conforme Lee e Tompkins (equação 6)

$C_{i}=$ Valores não observados - que são constantes no tempo e correlacionados com "q" - que impactam em inv

$T D C F=$ Taxa de despesa de Consumo das Famílias como variável de controle. Foram testadas quatro variáveis de controle: Produto Interno Bruto (PIB), Necessidade de Capacidade Líquida de Financiamento (CLF), Formação Bruta de Capital Fixo (FBCF) e Despesa de Consumo das Famílias (DCF) - essa última a que gerou melhores resultados e foi utilizada no modelo

$\varepsilon_{i t}=$ Erro aleatório que capta todas as outras dependências que não estão consideradas no modelo.

$i=$ Empresa, $i \in I:[1 ; 421]$

$t=$ Ano, $t \in I:[2002 ; 2008]$

A hipótese a ser testada é de que exista relação positiva entre o "q" de Tobin e o investimento, ou seja: $\operatorname{\delta inv} / \delta \mathrm{q}>0$. Logo $H_{0}: \beta_{l}=0$ e $H_{l}>0$.

Destaca-se que os investimentos devam decorrer de fatores internos das empresas, como capacidade de investimento, restrição de capital, entre outros, como também em função de fatores 
macroeconômicos, como, por exemplo, taxa de crescimento da demanda agregada. A não-inclusão desses elementos no modelo geraria erros de especificação, resultando em distorções do processo e estimação.

A exemplo dessas questões, Hennessy, Amnon e Whited (2007) realizaram um estudo onde constataram que além do "q" de Tobin também havia efeitos internos que determinavam o nível de investimentos. Em seus resultados, concluíram que considerar unicamente o "q" seria um erro, pois elementos como fluxo de caixa e restrição de capital também seriam significantes para explicar o investimento. Em um estudo com uma ampla amostra (1971 a 2004), Chang, Faff, Kwok e Wong (2009) também evidenciam a importância do fluxo de caixa e das restrições de capital na determinação do investimento, sendo que as empresas que não sofrem restrição de capital apresentam uma sensibilidade do investimento, em relação ao fluxo de caixa, significativamente superior àquelas que apresentam restrição.

Em relação aos fatores internos, não foram incluídas variáveis como as utilizadas pelos autores mencionados anteriormente em função de não se dispor do acesso a um banco de dados que permitisse construí-las e, ao mesmo tempo, em função de que se está testando a "validade" do "q" de Tobin para empresas brasileiras de capital aberto. Nesse caso, fatores internos serão captados pela variável " $C_{i}$ ". Dessa forma, reconhece-se aqui a existência desses fatores e que sua inclusão geraria resultados importantes, mas, ao mesmo tempo, crê-se que sua não-inclusão não afete a relação do "q" com o investimento.

No que se refere aos fatores externos, o elemento fundamental, segundo a percepção neo keynesiana, conforme Casagrande (2002b), seria a demanda agregada - em termos de tendência de crescimento. Seguindo essa lógica, é que se decidiu por incluir as variáveis de controle mencionadas anteriormente e com isso evitar um erro de especificação.

O modelo apresentado na equação (8) foi testado como versão inicial para as quatro formas de investimento estabelecidas. Cada uma delas foi cotejada com as duas formas de cálculo do "q" de Tobin. Esse procedimento gerou oito regressões iniciais, que depois foram modeladas para testar outras possíveis relações entre as variáveis.

A determinação do modelo de estimação, se efeitos fixos ou aleatórios, decorreu do teste de Hausman. Wooldridge (2002) explicita que esse teste é realizado sob a hipótese de estrita exogeneidade $-E\left(u_{i t} \mid x_{i}, C_{i}\right)=0 ; E\left(C_{i} \mid x_{i}\right)=E\left(C_{i}\right)=0$, onde as variâncias condicionais são constantes e a covariância é zero $-E\left(u^{\prime} u \mid x_{i}, c_{i}\right)=\sigma_{u}^{2} I_{T}$; e que $C_{i}$ é homoscedástico $\left(\operatorname{Var}\left(\mathrm{C}_{\mathrm{i}} \mid \mathrm{x}_{\mathrm{i}}\right)=\operatorname{Var}\left(\mathrm{C}_{\mathrm{i}}\right)\right.$.

Gujarati (2006) atribui que o uso de efeitos fixos ou aleatórios depende da pressuposição que se 
faz sobre a correlação entre o erro específico e as variáveis explicativas. Nesse sentido, crê-se que o melhor modelo seria o efeito fixo, pois a suposição é de que exista uma forte correlação entre os erros específicos e o "q". Além disso, o efeito aleatório é aconselhado quando se tem uma amostra aleatória dos dados, o que não foi o caso, reforçando assim, teoricamente, a indicação de efeitos fixos. No entanto, o teste de Hausman tem sido amplamente utilizado e aqui também foi realizado. Esse teste tem como hipótese nula que ambos os modelos não diferem. Uma vez rejeitada a hipótese nula, tem-se uma evidência a favor da regressão por efeitos fixos.

\section{População, Amostra e Fonte de Dados}

Para a pesquisa, foram selecionadas todas as empresas de capital aberto registradas na Bolsa de Valores de São Paulo - Bovespa, com dados disponíveis no sistema Economática. O período de estudo compreendeu os anos de 2002 a 2008, que são os anos mais recentes e que sofreram menos oscilações de mercado. Como nesse período ocorreu uma ampliação significativa das taxas de inflação e desvalorização cambial, em 2002, e a eclosão da crise a partir do suprime nos Estados Unidos, em 2008, estes foram incluídos e retirados das regressões para avaliar a estabilidade dos resultados.

Assim, a amostra contemplou sete anos com 421 empresas, cada um deles, resultando em 2.947 observações. Aquelas empresas que por-ventura não apresentaram as demonstrações contábeis em determinado ano, mas o fizeram em outros, constam da amostra. E aquelas cuja estimação do "q" de Tobin tenha apresentado valor negativo foram excluídas da amostra. Pela teoria, não existe um "q" negativo, já que essa variável é a relação entre o valor de mercado da empresa e seu custo de reposição. $\mathrm{O}$ valor de mercado foi obtido pelo somatório da multiplicação dos valores das ações ordinárias e preferenciais pelos seus respectivos valores, dados esses obtidos na base de dados da Economática. Após as exclusões, resultou uma amostra de 1.427 empresas.

A Despesa de Consumo das Famílias foi obtida no site do IBGE e foi considerada em valores constante a preços de 2008, inflacionados pelo IGPM.

\section{ANÁLISE DOS RESULTADOS}

Os valores negativos para o "q" de Tobin ocorreram quando o ativo circulante era maior que o valor de mercado da empresa somado ao capital de terceiros (passivo circulante mais exigível a longo prazo). Embora a metodologia proposta possa suprir a dificuldade de obtenção do cálculo do custo de 
reposição, atuando então como uma proxy para a relação de valor de mercado e custo de reposição do capital, apresenta a deficiência de gerar valores negativos, o que indica uma grave distorção dessa variável em determinadas situações. Esse problema decorre do baixo valor de mercado, que pode ser consequência de uma não-eficiência do mercado de capitais, que acaba não refletindo corretamente a real capacidade de geração de valor da empresa.

Realizando as regressões conforme indicado nos procedimentos metodológicos - equação (8) para os quatro formatos de investimentos e os dois do "q" de Tobin - os resultados foram insatisfatórios, gerando uma relação negativa entre o investimento e o "q". Os testes " $t$ " indicaram valores não significativamente diferentes de zero, isto é, p-valores para os " $t s$ " calculados muito superiores a 0,1 .

Em vista desses resultados, decidiu-se excluir as instituições financeiras da análise, por se tratar do setor de serviços em que as relações de custos de reposição de capital diferem das demais. Entretanto, o coeficiente angular permaneceu negativo. Por conta de essas estimações não serem significantes do ponto de vista estatístico e também do ponto de vista teórico, não se apresentam aqui esses resultados.

Em virtude da não-significância, optou-se por trabalhar a modelagem da equação (8). Assim, incluiu-se na análise, para evitar viés de especificação e com isso distorcer os planos da regressão, o investimento com a variável "q" defasada e elevada ao quadrado.

A defasagem justifica-se uma vez que, segundo Blanchard (2001, p. 347), as variações nos investimentos estão mais associadas às variações do mercado de ações do ano anterior. Esse fato pode ser consequência de as empresas levarem tempo para tomar decisões de investimento. No seu estudo, o autor concluiu que existe uma forte relação entre o investimento e o "q" de Tobin do ano anterior para as empresas dos Estados Unidos. No cálculo do "q" defasado denominado de q(-1), foram utilizados os dados do investimento do ano " $\mathrm{t}$ " relacionados com o "q" do ano anterior.

O valor de "q" ao quadrado $\left(\mathrm{q}^{2}\right)$ deve-se ao fato de se esperar que a influência do "q" nos investimentos seja positiva, mas que não cresça de forma constante, mas sim, que ela cresça a taxas decrescentes. Logo, o sinal do beta esperado para essa variável seria negativo.

Foram testadas como variáveis de controle Produto Interno Bruto (PIB), Despesas de Consumo Final das Famílias (DCF), Formação Bruta de Capital Fixo (FBCF) e Capacidade Líquida de Financiamento (CLF). Com exceção dessa última, todas as demais apresentaram problemas de raiz unitária, o que geraria uma regressão espúria caso fosse introduzida em nível. Mesmo em primeira ou segunda diferença, elas continuam não sendo estacionárias. Ao transformá-las em taxas, os testes de 
Levin, Lin \& Chu (2002), Im, Pesaran \& Shin "W-stat" (2003); ADF-Fisher e PP-Fisher (MADDALA e WU, 1999), indicaram não existência de raiz unitária (p-valores inferiores a 1\%).

A equação (9) foi o melhor resultado obtido das estimações com a modelagem da equação (8), tendo como investimento o IM, conforme mencionado anteriormente, e o "q" segundo Chung e Pruit (equação 5). Nesta, o teste de Hausman resultou em um $\chi^{2}$ de 10,5111, com quatro graus de liberdade, gerando uma probabilidade de 0,03 , indicando, ao nível de $5 \%$ de significância, a regressão a favor do efeito fixo. Logo a regressão foi realizada com efeitos fixos para empresas. Os anos não foram incluídos como fixos, pois estes não foram significantes em nenhuma regressão.

Destaca-se que o modelo utilizado por Hennessy, Amnon e Whited (2007), comentado anteriormente, também foi realizado com efeitos fixos, sendo que estes foram aplicados tanto para as empresas como para os anos.

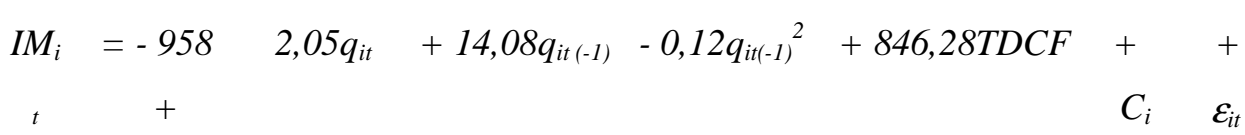

$\begin{array}{llllll}\text { p-valores de } & 0,006 & 0,067 & 0,000 & 0,001 & 0,007\end{array}$

“ $t$ "

Todas as variáveis foram significantes em nível de $1 \%$, exceto "q", que foi a $10 \%$. O teste de White não evidenciou problemas de heterocedasticidade (p-valor de 0,20) e o teste "d" de DurbinWatson (DW) ficou, marginalmente, na zona de indefinição com um valor de 1,622. A tentativa de estimar por quase diferenças para corrigir o problema de autocorrelação gerou uma ultrapassagem do valor do teste de DW piorando os resultados da estimação. O coeficiente de determinação indicou um grau razoável de explicação do modelo, de $17 \%$.

Com esses resultados, pode-se inferir que as empresas brasileiras de capital aberto, para o período analisado, tiveram um comportamento consistente com o que previa o modelo do "q" de Tobin. Esse efeito ocorre no mesmo ano, embora com menor impacto e menor significância do que no ano seguinte. Ainda, conforme esperado, seu efeito se dá a taxas decrescentes, conforme pode ser observado no sinal do beta para o valor do " $\mathrm{q}_{(-1)}$ " elevado ao quadrado.

Realizando o mesmo procedimento de estimação, porém usando INV, obteve-se o seguinte resultado: 


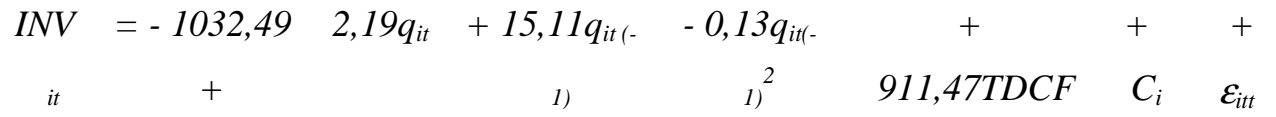

$\begin{array}{llllll}p \text {-valores de } & 0,006 & 0,06 & 0,000 & 0,001 & 0,007\end{array}$

Os resultados obtidos na equação (10) diferem marginalmente daqueles da equação (9). Os betas com uma ligeira diferença e os p-valores até a terceira decimal são idênticos. Destaca-se que esses dois resultados levaram em conta o investimento como variação percentual. Já as tentativas de relacionar investimento em nível (IMV e INVV) com o "q" não foram profícuas. Todas elas resultaram em dados não significantes.

Após esses testes, estimaram-se as relações do investimento com o "q" de Tobin conforme Lee e Tompkins (1999). Essa variável foi calculada da seguinte forma:

$q e=\frac{V M+E L P+E S T+P C-A C}{A T}$

Em que:

$q e=$ "q" de Tobin calculado conforme o procedimento proposto por Lee e Tompkins;

$V M=$ Valor de mercado das ações das empresas;

$E L P=$ Valor contábil do exigível a longo prazo;

$E S T=$ Valor contábil dos estoques;

$P C=$ Valor contábil do passivo circulante;

$A C=$ Valor contábil do ativo circulante;

$A T=$ Valor contábil dos ativos totais da empresa-

A diferença desse modelo para o proposto por Chung e Pruitt (1994), testado anteriormente, é o acréscimo do estoque no numerador, resultando num "q" maior. O resultado dessa estimação pode ser observado na equação (12). 


\begin{tabular}{|c|c|c|c|c|c|c|}
\hline & $\begin{array}{c}I M \\
{ }_{i t}\end{array}$ & $\begin{array}{c}=-955,59 \\
+\end{array}$ & $2,02 q_{i t}$ & $\begin{array}{c}+13,94 q e_{i t-(} \\
\text { 1) }\end{array}$ & $\begin{array}{c}-0,12 q e_{i t-} \\
1)^{2}\end{array}$ & $\begin{array}{c}+ \\
842,46 T D C \\
F\end{array}$ \\
\hline $\begin{array}{c}p \text {-valores de } \\
\text { "«” }\end{array}$ & & 0,007 & 0,070 & 0,000 & 0,002 & 0,008 \\
\hline
\end{tabular}

Ao se analisar a equação (12) ${ }_{2}$ pode-se perceber que seus parâmetros estão muito próximos dos obtidos na equação (9). Da mesma forma as demais estatísticas, como o DW e o coeficiente de determinação. Com isso pode-se afirmar que não há diferença em termos estatísticos ao estimar a regressão pelo método sugerido por Chung e Pruit (1994) ou por Lee e Tompkins (1999), a não ser por uma perda mínima de significância nos parâmetros estimados para esse último modelo.

Ao se estimar um modelo da equação (10), substituindo-se "q" por "qe", os resultados também são muito semelhantes. Ainda, não se obtiveram valores significativos com a estimação dos valores do investimento em nível com a utilização do "qe".

Com esses resultados pode-se afirmar, para o período analisado, que o "q" de Tobin é significativo para explicar a taxa de variação dos investimentos para as empresas de capital aberto no Brasil. Ainda, destaca-se que os parâmetros estimados permaneceram estáveis ao se excluir os anos de 2002 e de 2008, que apresentaram problemas de estabilidade da economia.

\section{CONSIDERAÇÕES FINAIS}

Pelos testes, pôde-se concluir que o modelo teórico proposto por Tobin pode ser considerado um elemento importante na decisão dos investimentos das empresas e que a utilização dos dados contábeis, como uma aproximação do custo de reposição, possui relevância, além de apresentar um avanço no que concerne à facilidade de obtenção dos dados.

É preciso destacar que, em termos de modelagem, a inclusão da defasagem de um período do “q" e seu valor ao quadrado foi o que possibilitou os resultados esperados do modelo em termos teóricos e com parâmetros significativos. A existência dessa defasagem pode ser decorrência de as empresas demorarem certo tempo para tomar suas decisões de investimentos e estes crescem a taxas decrescentes. 
Os resultados dos testes comprovam a relevância da contabilidade para explicar agregados econômicos e a possibilidade de predição de investimentos através de análises com dados contábeis, o que representa um grande avanço, pois esses dados são obtidos facilmente nas demonstrações contábeis das empresas.

Destaca-se que não foram incorporados na análise os fatores internos que influem na decisão de investir conforme determinado por outros pesquisadores, mencionados no texto. Essa exclusão decorreu da não-disponibilidade de acesso dos autores a um banco de dados, como o Comustat da Standard \& Poor, que permitisse a construção dessas variáveis, sendo que este é um aspecto interessante a ser testado nas empresas brasileiras.

Outra possibilidade de análise seria calcular o valor de mercado das empresas através da utilização de algum método de avaliação de empresas (Modelo de Gordon, por exemplo) e usar esse valor para o cálculo do "q" de Tobin. A comparação desses resultados seria importante para avaliar os processos de aproximação do valor de reposição do capital.

\section{REFERÊNCIAS}

ANDRADE, P. C. O comportamento do investimento agregado no Brasil - 1960-1987. 1987. $191 \mathrm{f}$. Dissertação de Mestrado em Economia, Departamento de Economia, Pontifícia Universidade Católica do Rio de Janeiro, Rio de Janeiro, 1987.

BLANCHARD, O. Macroeconomia: teoria e política econômica. Rio de Janeiro: Campus, 2001.

CASAGRANDE, E. E. Modelos de investimento: metodologia e resultados. Revista de Economia Política, v. 22, n. 1, p. 85, 2002a.

CASAGRANDE, E. E. As teorias keynesianas do investimento. São Paulo: Cultura Acadêmica, 2002b.

CHANG, X; FAFF, R; KWOK, W. C; WONG, G. Financial constraints, mispricing and corporate investment. Working Paper Series, 12 Mar. 2009. Disponível em http://papers.ssrn.com/sol3/papers.cfm?abstract_id=1101361. Acesso em 21.07.2009. 
ARTIGOS - ANÁLISE DA CAPACIDADE EXPLICATIVA DO INVESTIMENTO PELO “q” DE TOBIN EM EMPRESAS BRASILEIRAS DE CAPITAL ABERTO

Edson Luis Kammler - Tiago Wickstrom Alves

CHUNG, K. H; PRUITT, S. W. A simple approximation of Tobin's q. Financial Management, v. 23, n. 3, p. 70-74, 1994.

DORNBUSCH, R; FISCHER, S. Macroeconomia. São Paulo: McGraw-Hill do Brasil, 1982.

HENNESSY, C. A; LEVY, A; WHITED, T. M. Testing Q theory with financing frictions. Journal of Financial Economics. v. 83, n. 3, p. 691-717, 2007.

ERICKSON, T; WITHED, T. M. Measurement error and the relationship between investment and q. Journal of Political Economy, v. 108, n. 5, F, 2000.

FAMA, R; BARROS, L. B. C. Q de Tobin e seu uso em finanças: aspectos metodológicos e conceituais. Caderno de Pesquisas em Administração. São Paulo, v. 7, n. 4, p. 27-43, 2000.

GORDON, R. J. Macroeconomia. 7. ed. Porto Alegre: Bookman, 2000.

GUJARATI, D. Econometria Básica. 4. ed. Rio de Janeiro: Campus, 2006.

IM, K. S; PESARAN, M. H; SHIN, Y. Testing for unit roots in heterogeneous panels. Journal of Econometrics, v. 115, p. 53-74, 2003.

KASSAI, J. R; KASSAI, S; ASSAF NETO, A. Índice de Especulação de Valor Agregado - IEVA. Revista de Contabilidade \& Finanças, n. 30, p. 32-45, 2002.

LEE, D. E; TOMPKINS, J. G. A modified version of the Lewellen and Badrinath of Tobin's q. Financial Management, v. 28, n. 1, p. 20-31, 1999.

LEVIN, A; LIN, C. F; CHU, C. Unit root tests in panel data: asymptotic and finite-sample properties. Journal of Econometrics, v. 108, p. 1-24, 2002.

LINDENBERG, E. B; ROSS, S. A. Tobin's q ratio and industrial organization. The Journal of Business, v. 54, n. 1, 1981. 
ARTIGOS - ANÁLISE DA CAPACIDADE EXPLICATIVA DO INVESTIMENTO PELO “q” DE TOBIN EM EMPRESAS BRASILEIRAS DE CAPITAL ABERTO

Edson Luis Kammler - Tiago Wickstrom Alves

MADDALA, G. S; WU, S. A comparative study of unit root tests with panel data and a new simple test. Oxford Bulletin of Economics and Statistics, v. 61, p. 631-652, 1999.

PAULO, E. Capital intelectual: formas alternativas de mensuração. In: CONGRESSO BRASILEIRO DE CONTABILIDADE, 16, 2000, Goiânia. Anais. Goiânia: CBC, 2000.

SACHS, J. D.; LARRAIN, B. F. Macroeconomia. São Paulo: Makron Books, 1995.

SHAPIRO, E. Análise macroeconômica. 2. ed. São Paulo: Atlas, 1985.

TOBIN, J. A general equilibrium approach to monetary theory. Journal of Money, Credit and Banking, v. 1, n. 1, p. 15-29, 1969.

WOOLDRIDGE, J. The econometrics of cross section and panel data. Cambridge: MIT Press, 2002. 